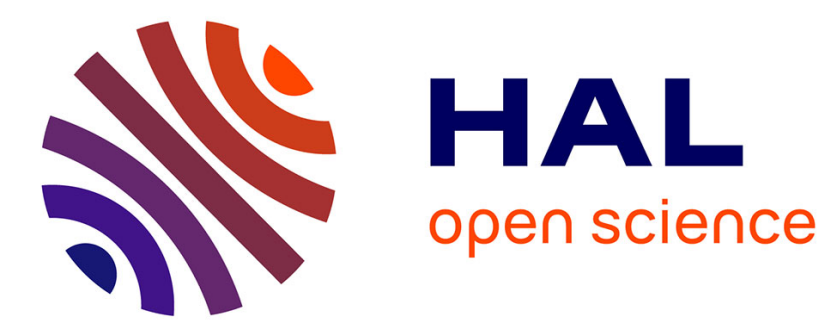

\title{
Ultrafast double-quantum NMR spectroscopy
}

Adrien Le Guennec, Patrick Giraudeau, Stefano Caldarelli, Jean-Nicolas

Dumez

\section{To cite this version:}

Adrien Le Guennec, Patrick Giraudeau, Stefano Caldarelli, Jean-Nicolas Dumez. Ultrafast double-quantum NMR spectroscopy. Chemical Communications, 2015, 51 (2), pp.354-357. $10.1039 / \mathrm{c} 4 \mathrm{cc} 07232 \mathrm{~d}$. hal-02142148

\section{HAL Id: hal-02142148 \\ https://hal.science/hal-02142148}

Submitted on 13 Nov 2020

HAL is a multi-disciplinary open access archive for the deposit and dissemination of scientific research documents, whether they are published or not. The documents may come from teaching and research institutions in France or abroad, or from public or private research centers.
L'archive ouverte pluridisciplinaire HAL, est destinée au dépôt et à la diffusion de documents scientifiques de niveau recherche, publiés ou non, émanant des établissements d'enseignement et de recherche français ou étrangers, des laboratoires publics ou privés. 


\section{COMMUNICATION}

\section{Ultrafast double-quantum NMR spectroscopy}

Cite this: DOI: 10.1039/x0xx00000x

\author{
Adrien Le Guennec, ${ }^{\mathrm{a}, \mathrm{b}}$ Patrick Giraudeau, ${ }^{\mathrm{b}, \mathrm{c}}$ Stefano Caldarelli ${ }^{\mathrm{a}, \mathrm{d}}$ and Jean-Nicolas \\ Dumez ${ }^{\mathrm{a} *}$
}

Received 00th January 2012,

Accepted 00th January 2012

DOI: $10.1039 / \times 0 \times x 00000 x$

www.rsc.org/
We demonstrate the acquisition of double-quantum NMR spectra in less than three seconds and illustrate the synergies between double-quantum and ultrafast NMR spectroscopy for the analysis of complex mixtures.

Among the most intriguing and challenging samples presented to the analyst, mixtures of molecules are of broad interest to many fields of chemistry. In many instances, purification is either prohibitively time-consuming, or impossible without alteration of the sample. Multidimensional nuclear magnetic resonance (nD NMR) spectroscopy is an essential tool for the analysis of such complex chemical systems, thanks to its superior resolution compared to 1D NMR. The use of $\mathrm{nD}$ NMR is expanding in cases such as metabolomics or natural products research, to name relevant examples. ${ }^{1}$ The acquisition of $\mathrm{nD}$ NMR spectra according to the original Jeener-Ernst scheme is impeded, however, by the need to sample parametrically one or more evolution dimensions. ${ }^{2}$ The resulting experimental times can be exceedingly long and impractical for the analysis of fast processes or of very large numbers of samples.

Ultrafast NMR (UFNMR) is a powerful approach to accelerate $\mathrm{nD} \mathrm{NMR}^{3}$ It relies on a spatial rather than temporal dimension to encode NMR interactions; using a magnetic resonance imaging (MRI)-inspired acquisition scheme, a full multidimensional spectrum can be obtained in a single scan. The unique potential of spatial encoding is a source of development in many areas of chemistry. Ultrafast versions of sequences such as COSY, TOCSY and HSQC have been used to study complex mixtures with significantly reduced experimental times. ${ }^{4}$ Further simplifications may be obtained by the use of other correlation strategies such as multidimensional multiple-quantum $\mathrm{NMR},{ }^{5}$ which has a lower number of signals compared to single-quantum experiments. For example, proton homonuclear double-quantum correlation spectra are free of auto-correlation ("diagonal") peaks, which can complicate the analysis of crowded COSY spectra. ${ }^{6}$ The extreme example is maximum-quantum spectroscopy, ${ }^{7}$ in which the MQ dimension is simplified drastically, thus allowing to differentiate between structurally similar compounds in a mixture. Proton doublequantum spectroscopy has been proposed for efficient water suppression, associated to diffusion filters, ${ }^{8}$ but it has also been shown to be suitable for quantitative analyses, again gaining benefit from the absence of diagonal peaks and of the associated " $t_{1}$-noise". 9

In this communication, we introduce an approach for ultrafast double-quantum NMR spectroscopy (ufDQS). We outline the principles of the spatial encoding of double-quantum frequencies and of their subsequent detection in ultrafast spectra. We then demonstrate the ufDQS sequence with the acquisition of a doublequantum spectrum of sucrose in less than $3 \mathrm{~s}$. We finally apply the ufDQS methodology to the analysis of a synthetic mixture of metabolites. The proposed approach combines the analytical strength of homonuclear multiple-quantum spectroscopy with the speed and flexibility of spatial encoding and exploits further synergies between the two concepts.

a
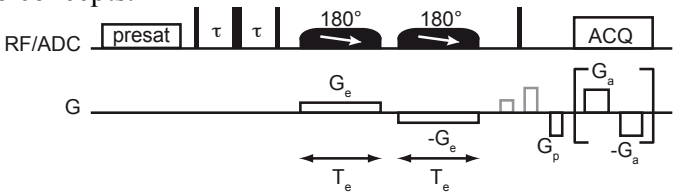

b

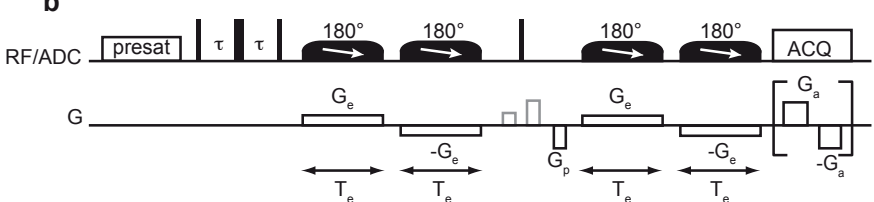

Fig. 1 Pulse sequences for ultrafast double-quantum NMR spectroscopy with a classic or $\mathbf{b}$ delayed acquisition. Gradients for spatial encoding and for coherence selection are shown in black and in grey, respectively. Hard $90^{\circ}$ and $180^{\circ}$ pulses are shown with thin and thick vertical bars, respectively. The last (mixing) has a duration corresponding to a $120^{\circ}$ flip angle. The delay $\tau$ corresponds to the build-up of anti-phase magnetisation. The presaturation module is optional and is used here for water suppression.

The NMR pulse sequence for ultrafast double-quantum spectroscopy (ufDQS) is shown in Fig. 1a. As the conventional DQS sequence, ${ }^{10}$ shown in Fig. S1, the UF sequence contains a spin-echo block flanked by $90^{\circ}$ pulses to generate multiple-quantum coherences. It 
also relies on a gradient pair around the mixing pulse to select double-quantum coherences in the evolution period. The evolution period contains a spatial encoding module, which consists of a pair

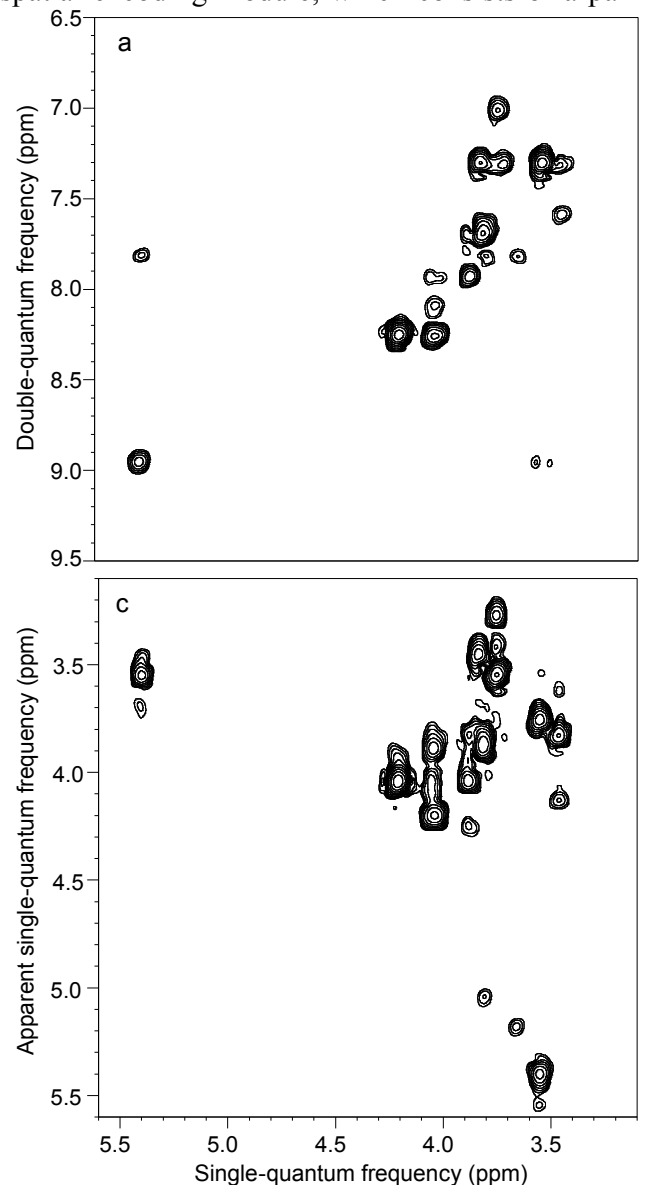

of frequency swept pulses that are played together with gradients of opposite signs.
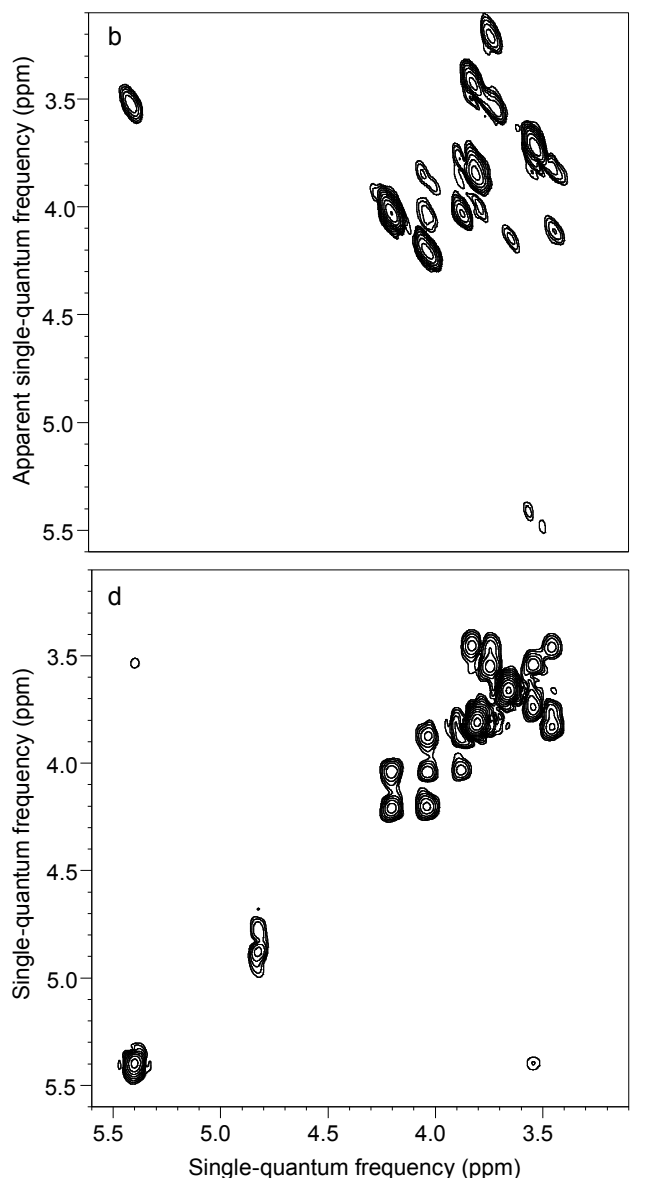

Fig. 2 Ultrafast NMR spectra of $0.1 \mathrm{M}$ sucrose in $\mathrm{D}_{2} \mathrm{O}$ : a DQS (sequence shown in Fig. 1a); c DQSsy (sequence shown in Fig. 1b); d COSY. All spectra were recorded in a single scan ( $2.2 \mathrm{~s}$ including water presaturation), using a double-quantum build-up delay $\tau=25 \mathrm{~ms}$. Spectrum $\mathbf{b}$ was obtained from spectrum $\mathbf{a}$ by a shearing transformation at a processing stage. All spectra were recorded with a $600 \mathrm{MHz}$ spectrometer equipped with a cryogenic probe.

Spatial encoding applies a phase shift:

$$
\boldsymbol{\phi}=\boldsymbol{C}\left(\boldsymbol{\Omega}_{\boldsymbol{i}}+\boldsymbol{\Omega}_{\boldsymbol{j}}\right) \mathbf{z}
$$

to double-quantum coherences of the form $I_{i+} I_{j+}, \Omega_{\mathrm{j}}$ is the chemical-shift offset of spin $i, z$ is the position along the vertical axis and $C$ is a proportionality constant that depends on the choice of the spatial encoding module. ${ }^{3 c, 11}$ The acquisition consists of an echo planar spectroscopic imaging (EPSI)-style block, analogous to the ufCOSY sequence, which has been analysed in great details. ${ }^{3 \mathrm{c}}$ The key difference with ufCOSY is that, because double rather than single-quantum frequencies are spatially encoded, the gradient echoes observed in the EPSI acquisition block have a position proportional to the sum of two chemical-shift offsets. In practice, the ufDQS sequence can be set up similarly to the ufCOSY sequence and available processing tools are suitable to the new sequences. ${ }^{12}$ The encoding strategy proposed here is complementary to the scheme of Walls and coworkers to read MQ frequencies in a single scan. ${ }^{13}$

Figure 2a shows a double-quantum NMR spectrum recorded in a single scan for a solution of sucrose. The acquisition time for this experiment was $163 \mathrm{~ms}$, resulting in a total experimental duration of $2.2 \mathrm{~s}$ including water presaturation. This further illustrates the ability of spatial encoding to yield few-second acquisition of arbitrary spectra. Double-quantum spectra may look unfamiliar compared to the more widespread COSY spectra, since cross-peaks connect two chemical shifts in the single-quantum dimension with their sum frequency in the
DQ axis. To facilitate the readout and the comparison with COSY, a shearing transformation may be performed. Figure $2 b$ shows the result of such a shearing transformation applied to the ufDQS spectrum of sucrose; an ultrafast COSY spectrum is also shown in Fig. 2d for comparison. Both spectra show complex lineshapes, a common feature in ultrafast NMR. ${ }^{14}$ While the cross-peaks are identical in the COSY and DQS spectrum, there are no diagonal peaks in the DQS case. The additional peaks observed in the DQS spectrum correspond to "relay peaks", and can be observed in conventional and UFNMR spectra. While they may complicate the analysis, they are also an additional source of structural information, ${ }^{6}$ for example in the study of small proteins. ${ }^{15}$

In conventional double-quantum spectroscopy, COSYlike spectra can also be obtained, with no need for a shearing transformation, by using a delayed acquisition. ${ }^{16}$ An evolution period is added to the DQS sequence between the mixing pulse and the acquisition. This approach can also be extended to the spatially encoded DQ spectra, yielding "symmetric" DQ spectra (DQSsy). As shown in Fig. 1b, this is achieved by adding a second spatial encoding module after the mixing pulse. This applies an additional phase shift:

$$
\boldsymbol{\phi}=-\boldsymbol{C} \boldsymbol{\Omega}_{\boldsymbol{i}} \boldsymbol{z}
$$

to coherences of the form $I_{i} I_{i z}$. As a result, the positions of the echoes in the spatially encoded dimension are given by a single rather than double-quantum frequency. The spectrum obtained 
with this pulse sequence is shown in Fig. 2c. While this strategy has the advantage that it does not require a shearing transformation during post-processing, the second spatial encoding block can increase signal losses due to translational molecular diffusion or lead to the presence of additional artefacts, so that the use of the non-delayed acquisition is recommended
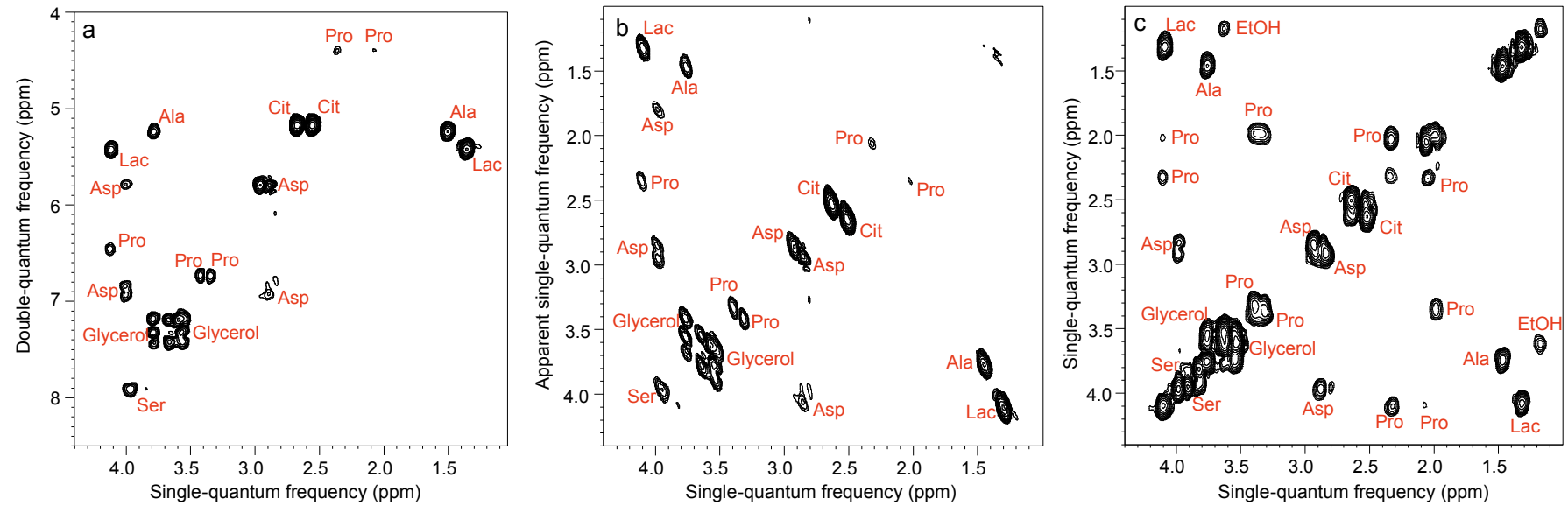

Fig. 3 Ultrafast NMR spectra of a mixture of 7 metabolites in $\mathrm{H}_{2} \mathrm{O} / \mathrm{D}_{2} \mathrm{O}(90 / 10)$ : a DQS (sequence shown in Fig. 1a); c COSY. All spectra were recorded in four interleaved scans to increase the observable spectral width, and four dummy scans resulting in an acquisition time of $41 \mathrm{~s}$, using a double-quantum buildup delay $\tau=25 \mathrm{~ms}$. Spectrum $\mathbf{b}$ was obtained from spectrum a by a shearing transformation at a processing stage. All spectra were recorded with a $600 \mathrm{MHz}$ spectrometer equipped with a cryogenic probe.

Note that an alternative 2-scan pulse sequence has been proposed recently to obtain "diagonal-free COSY" spectra, ${ }^{17}$ and can be compared against the ufDQS sequence in an application-specific manner.

In addition to providing correlation information, multidimensional spectroscopy has additional benefits when the complexity of $1 \mathrm{D}$ spectra is too high to disentangle individual peaks. A key application of 2D NMR is the analysis of complex mixtures, ${ }^{1}$ the spectra of which consist of multiple overlapping peaks. Figure $3 \mathrm{a}$ shows a double-quantum spectrum for a synthetic mixture of metabolites, obtained with four "interleaved" scans. In this case, the absence of diagonal peaks is essential to identify cross-peaks that lie close to the diagonal, such as the cross peaks for citrate or serine. The lower density of peaks of the double-quantum approach makes it appealing for spatially encoded spectra, which usually have a lower resolution than their conventional counterparts.

Originally proposed in ultrafast MRI, interleaving is a general approach to increase the spectral widths in ultrafast 2D NMR, at the cost of recording several scans. ${ }^{3 \mathrm{~d}}$ Experimental durations still remain far shorter than for conventional acquisitions. Phase inconsistencies may lead to artefacts but several strategies have been developed to identify and remove them. ${ }^{18}$ In addition, these "ghost" peaks are most noticeable in the presence of very intense peaks; in the double-quantum spectrum, the absence of auto-correlation peaks reduces the impact of ghost peaks.

The spectra obtained in Figure 3 demonstrate the potential of ufDQS for the analysis of metabolic mixtures. The potential of conventional DQ spectroscopy for the quantitative analysis of biological samples has been recently demonstrated, ${ }^{19}$ and the acquisition strategy described here will certainly form a powerful analytical tool for such applications. Ultrafast NMR typically yields spectra with limited resolution ${ }^{20}$ and the simplified double-quantum spectra are beneficial in this respect. Still, the speed obtained with spatial encoding comes at a price in sensitivity. ${ }^{3 c, 4}$ The concentrations for metabolites in the mixture studied here ranged from 13 to $27 \mathrm{mM}$. While the concentrations are relatively high in this synthetic mixture, hybrid strategies that consist of averaging multiple spatially encoded spectra ${ }^{20}$ have been shown to be useful for application such as quantitative $\mathrm{NMR}^{21}$ or metabolomics, where samples are much less

concentrated. ${ }^{22}$. While double-quantum coherences are predicted to have shorter relaxation times, the encoding duration employed here did not lead to systematic losses. The heterogeneity of homonuclear J-couplings leads to the non-ideal excitation of specific double-quantum coherences and this can be addressed, e.g., by averaging multiple scans with varying build-up times. ${ }^{23}$ Some correlation peaks are more intense in ufCOSY while some are more intense in ufDQS (see SI); the detailed analysis of peak volumes in ultrafast spectra is a complex and ongoing study.

We have introduced two new pulse sequences for ultrafast double-quantum NMR spectroscopy. Using spatial encoding, DQS spectra can be obtained in one or a few scans. The absence of auto-correlation peaks in the DQS spectra simplifies the analysis of cross-peaks that are close to the diagonal and is also beneficial when interleaving is employed to increase the spectral width in spatially encoded experiments. The ufDQS approach -optionally in a multi-scan implementation- will prove useful for applications of ultrafast NMR, such as the quantitative analysis of complex mixtures, which can have a large dynamic range of concentration. The spatial encoding of multiple-quantum frequencies may also be generalised to higher orders. Research along these avenues is currently in progress.

P. Giraudeau acknowledges funding from the "Agence Nationale de la Recherche" for young researchers (ANR Grant 2010-JCJC-0804-01), as well as Laura Castañar (University of Barcelona) for preliminary investigations.

\section{Notes and references}

${ }^{a}$ Institut de Chimie des Substances Naturelles, CNRS UPR 2301, Avenue de la Terrasse 91190 Gif-sur-Yvette, France

E-mail: jeannicolas.dumez@cnrs.fr

${ }^{b}$ Université de Nantes, CNRS, CEISAM UMR6230, BP92208, 2, rue de la Houssinière, F-44322 Nantes Cedex 03, France

${ }^{c}$ Institut Universitaire de France, 103 Boulevard St. Michel,75005 Paris Cedex 5, France.

${ }^{d}$ Aix Marseille Université, Centrale Marseille, CNRS, iSm2 UMR 7313, 13397 Marseille, France.

$\dagger$ Electronic Supplementary Information (ESI) available: i/ Materials and Methods; ii/ supplementary figures with conventional 2D NMR experiments; iii/ comparison of SNR for the ufCOSY and 
ufDQS sequences iv/ pulse program for the ufDQS sequence. See DOI: $10.1039 / \mathrm{c} 000000 \mathrm{x} /$

1 (a) S. L. Robinette, R. Brueschweiler, F. C. Schroeder and A. S. Edison, Accounts of Chemical Research, 2012, 45; (b) K. Bingol and R. Brueschweiler, Analytical Chemistry, 2014, 86, 47.

2 R. R. Ernst, G. Bodenhausen and A. Wokaun, Principles of Nuclear Magnetic Resonance in One and Two Dimensions, Oxford University Press, Oxford, 1990.

3 (a) L. Frydman, T. Scherf and A. Lupulescu, Proceedings of the National Academy of Sciences of the United States of America, 2002, 99, 15858; (b) M. Mishkovsky and L. Frydman, Annual Review of Physical Chemistry, 2009, 60, 429; (c) A. Tal and L. Frydman, Progress in Nuclear Magnetic Resonance Spectroscopy, 2010, 57, 241; (d) L. Frydman, A. Lupulescu and T. Scherf, Journal of the American Chemical Society, 2003, 125, 9204.

4 P. Giraudeau and L. Frydman, Annual Review of Analytical Chemistry, 2014, 7, 129.

5 (a) A. Wokaun and R. R. Ernst, Chemical Physics Letters, 1977, 52, 407; (b) Molecular Physics, 1979, 38, 1579; (c) G. Bodenhausen, Progress in Nuclear Magnetic Resonance Spectroscopy, 1980, 14, 137; (d) T. J. Norwood, Progress in Nuclear Magnetic Resonance Spectroscopy, 1992, 24, 295; (e) L. Braunschweiler, G. Bodenhausen and R. R. Ernst, Molecular Physics, 1983, 48, 535.

6 C. Dalvit and J. M. Bohlen, Annu. Rep. NMR Spectrosc., 1999, 37, 203.

7 G. N. Manjunatha Reddy and S. Caldarelli, Analytical Chemistry, 2010, 82, 3266.

8 (a) C. Dalvit and J. M. Bohlen, Journal of Magnetic Resonance Series B, 1996, 113, 195; (b) Nmr in Biomedicine, 1997, 10, 285.
9 E. Martineau, P. Giraudeau, I. Tea and S. Akoka, Journal of Pharmaceutical and Biomedical Analysis, 2011, 54, 252.

10 T. H. Mareci and R. Freeman, Journal of Magnetic Resonance, 1983, $\mathbf{5 1}, 531$.

11 P. Pelupessy, Journal of the American Chemical Society, 2003, 125.

12 M. Pathan, B. Charrier, I. Tea, S. Akoka and P. Giraudeau, Magnetic Resonance in Chemistry, 2013, 51, 168.

13 C. D. Ridge, L. Borvayeh and J. D. Walls, J. Chem. Phys., 2013, 138.

14 B. Shapira, A. Lupulescu, Y. Shrot and L. Frydman, Journal of Magnetic Resonance, 2004, 166, 152.

15 G. Wagner and E. R. P. Zuiderweg, Biochemical and Biophysical Research Communications, 1983, 113, 854.

16 D. L. Turner, Journal of Magnetic Resonance, 1982, 49, 175.

17 A. Banerjee and N. Chandrakumar, Journal of Chemical Physics, 2014, 140, 31103.

18 L. Rouger, B. Charrier, M. Pathan, S. Akoka and P. Giraudeau, Journal of Magnetic Resonance, 2014, 238, 87.

19 E. Martineau, I. Tea, S. Akoka and P. Giraudeau, NMR in Biomedicine, 2012, 25, 985.

20 M. Pathan, S. Akoka, I. Tea, B. Charrier and P. Giraudeau, Analyst, 2011, 136, 3157.

21 A. Le Guennec, I. Tea, I. Antheaume, E. Martineau, B. Charrier, M. Pathan, S. Akoka and P. Giraudeau, Analytical Chemistry, 2012, 84, 10831.

22 A. Le Guennec, P. Giraudeau and S. Caldarelli, Analytical Chemistry, 2014, 86, 5946.

23 G. N. M. Reddy and S. Caldarelli, Magnetic Resonance in Chemistry, 2013, 51, 240. 